\title{
Balkanologie
}

Balkanologie Revue d'études pluridisciplinaires

Vol. I, n 2 | 1997

Volume I Numéro 2

\section{Privatisation, démocratisation ou oligarchisation de la Bulgarie postcommuniste}

\section{Miroslav K. Popov et Elka N. Todorova}

Traducteur : Patrick Michels

\section{OpenEdition \\ Journals}

Édition électronique

URL : http://journals.openedition.org/balkanologie/219

DOI : 10.4000/balkanologie.219

ISSN : 1965-0582

Éditeur

Association française d'études sur les Balkans (Afebalk)

Édition imprimée

Date de publication : 1 décembre 1997

ISSN : 1279-7952

Référence électronique

Miroslav K. Popov et Elka N. Todorova, « Privatisation, démocratisation ou oligarchisation de la Bulgarie postcommuniste », Balkanologie [En ligne], Vol. I, n² 2 | 1997, mis en ligne le 02 juin 2008, consulté le 17 décembre 2020. URL : http://journals.openedition.org/balkanologie/219 ; DOI : https:// doi.org/10.4000/balkanologie.219

Ce document a été généré automatiquement le 17 décembre 2020.

(c) Tous droits réservés 


\title{
Privatisation, démocratisation ou oligarchisation de la Bulgarie postcommuniste
}

\author{
Miroslav K. Popov et Elka N. Todorova \\ Traduction : Patrick Michels
}

\section{NOTE DE L'ÉDITEUR}

Cet article a été présenté à la conférence internationale « Transition to What ? The Implications of Privatization in Eastern Europe, the European Union, the Balkans and Beyond », Komotini (Grèce), qui s'est déroulée du 26 juin au 2 juillet 1995. Traduit de l'anglais par Patrick Michels.

1 Depuis 1989, la Bulgarie a connu des changements radicaux. La législation s'est améliorée, une nouvelle constitution démocratique a été adoptée, l'établissement des institutions démocratiques progresse, tout comme la promotion des Droits de l'Homme.

2 Toutefois, la situation économique s'est significativement détériorée. Le PNB (produit national brut) du pays est inférieur de $25 \%$ à celui de 1990. La production industrielle l'est de $50 \%$. La pauvreté et le chômage se sont généralisés. La Bulgarie a aussi une dette extérieure élevée.

Dans la plupart des analyses, la privatisation en Bulgarie est perçue comme un double processus de dénationalisation de la propriété d'État et des entreprises municipales. La "Loi pour la transformation et la privatisation des entreprises étatiques et municipales» a été adoptée le 23 avril 1992. Officiellement, le processus de privatisation a débuté en mai 1993, lorsque s'est effectué le premier gros transfert d'actions.

4 Les privatisations les plus courantes se réduisent à un transfert rapide des entreprises publiques vers le secteur privé dans l'espoir que celui-ci sera plus efficace dans leur réorganisation ou à un transfert après leur réorganisation. De manière générale, les 
pays se réformant (dont la Bulgarie) ont choisi la première solution. Mais la vitesse des transactions effectuées se révèle plus lente que prévue. En Hongrie, en 1995, 40 \% seulement des entreprises publiques étaient vendues, les industries chimiques, pharmaceutiques et énergétiques étant demeurées propriétés publiques. En Pologne, seulement 2500 des 8400 entreprises l'étaient. En Macédoine, 200 entreprises. En Grèce, toutes les grandes compagnies, gérant l'énergie électrique, le téléphone ou la construction navale, sont encore sous le contrôle absolu de l'État ${ }^{1}$.

\section{La situation économique en Bulgarie}

5 L'économie nationale a été libéralisée selon les préceptes des institutions économiques internationales. Pendant ces cinq années, la législation a été renouvelée et les structures de propriété ont été radicalement modifiées (certain secteurs de l'économie, tels que le commerce intérieur et la construction, sont déjà soumis, de façon prédominante, à l'initiative privée). La part du secteur privé dans le PNB a augmenté de manière significative.

6 Au printemps 1994, la monnaie nationale a été sensiblement dévaluée. L'écart entre le taux d'inflation de $45 \%$ prévu dans le budget annuel, établi en accord avec le Fonds Monétaire International, et le taux réel d'inflation à la fin de l'année de 121,9\% s'est ainsi creusé. Avec son niveau de PNB per capita en 1993 (1276), la Bulgarie est plus proche du groupe des 77 pays en voie de développement établi par l'ONU que de celui des pays industrialisés ${ }^{2}$. La chute du PNB, aussi bien que celle de la production industrielle, ont atteint des niveaux pratiquement inconnus en temps de paix.

7 Les déficits du budget de l'état sont chroniques. À la fin de l'année 1994, la dette intérieure de l'État s'élevait à 277,2 milliards de levs, soit la moitié du PNB bulgare (500 milliards de levs).

8 Les investissements étrangers sont plutôt limités. À la fin de 1994, ils représentaient 467,2 millions de dollars. On estime à 150 millions de dollars le montant des investissements additionnels, liés aux projets de privatisation. Celui des investissements indirects se monterait à 120 millions de dollars. Les investisseurs les plus importants sont l'Allemagne, avec 178 millions de dollars, les Pays-Bas (57,5 millions de dollars) et la Suisse (50,5 millions de dollars). En ce qui concerne les projets d'investissement, la Grèce se place en tête avec 573 projets estimés à 33 millions de dollars. La moyenne des investissements enregistrés des compagnies étrangères est de 1000 dollars $^{3}$.

Tableau 1 : résultats économiques comparés

\begin{tabular}{|l|r|r|r|r|r|r|}
\hline \multirow{2}{*}{ Pays } & \multicolumn{3}{|c|}{ Croissance du PNB en \% } & \multicolumn{4}{|c|}{ Inflation (par année) } \\
\hline & 1992 & 1993 & 1994 & 1992 & 1993 & 1994 \\
\hline Albanie & $-9,7$ & 11,0 & 8,0 & 236,5 & 30,9 & 24,1 \\
\hline Bulgarie & $-6,0$ & $-4,2$ & 0,0 & 83,0 & 73,0 & 96,0 \\
\hline Rép. tchèque & $-7,1$ & $-0,3$ & 2,0 & 12,7 & 18,2 & 9,0 \\
\hline
\end{tabular}




\begin{tabular}{|l|r|r|r|r|r|r|}
\hline Hongrie & $-4,5$ & $-2,3$ & 1,0 & 21,6 & 21,1 & 21,1 \\
\hline Pologne & 1,0 & 3,8 & 4,5 & 44,3 & 37,7 & 28,0 \\
\hline Roumanie & $-13,8$ & 1,0 & 1,0 & 204,4 & 255,2 & 145,0 \\
\hline Russie & $-19,0$ & $-12,0$ & $-12,0$ & 1350,0 & 930,2 & - \\
\hline
\end{tabular}

Source : « La Bulgarie à la lumière de l'économie mondiale », Kontinent, 19/01/95

9 Après cette brève description de la situation économique de la Bulgarie, qu'en est-il du processus de privatisation?

\section{Le processus de privatisation (traits généraux)}

10 La privatisation en Bulgarie doit s'apprécier selon la stratégie de privatisation et la rapidité des transferts des entreprises dont le capital est détenu par l'État ou la commune (selon la «Loi pour la transformation et la privatisation des entreprises étatiques et municipales $»^{4}$ ); selon la privatisation des terres et des anciennes fermes collectives ; et selon le succès de la restitution (d'après les lois de restitution).

\section{La restitution}

11 Elle a été ouvertement critiquée. Elle a couvert toutes les habitations, parcelles de terrain et jardins à hauteur de $64,2 \%$ de ce qui était réclamé. Plus de la moitié des réclamations ont déjà été satisfaites. Les retards dans la restitution sont dus aux conflits de propriété, aux problèmes de compensation de ce qui n'existe plus.

La restitution, en tant que telle, a joué un rôle particulier dans le changement de propriété. À la fin de 1994, des biens pour une valeur de 10,3 milliards de levs ont été rendus à leurs anciens propriétaires, c'est-à-dire l'équivalent de $2 \%$ du PNB. D'après certaines analyses économiques ${ }^{5}$, le processus de restitution est pratiquement terminé en ce qui concerne les petites et moyennes entreprises. On peut, de ce fait, la considérer comme une forme de privatisation à petite échelle. Elle a créé les conditions nécessaires à la formation d'un vrai marché de l'immobilier, de la libre entreprise et au développement du secteur privé.

13 Toutefois, malgré l'impact positif de la restitution sur le développement du marché, la plupart des problèmes insolubles qu'elle pose entraînent des effets négatifs quant à la privatisation des entreprises publiques (étatiques ou municipales). La restitution ne retarde pas seulement la privatisation, souvent elle l'entrave.

Dans le même temps, de nombreux centres sociaux et culturels (cinémas, théâtres) et de service (crèches, maisons de retraite) ont fermé, provoquant opposition et critiques.

La restitution a accru l'inégalité des revenus. Les $20 \%$ les plus riches percevaient $24 \%$ du revenu en 1991 et $39 \%$ en 1993, tandis que les $20 \%$ les plus pauvres en recevaient $10 \%$ en 1991 et $8 \%$ en 1993. Les deux tiers de la population vivent avec un revenu inférieur au minimum vital. 


\section{La privatisation des terres}

16 restituées en de brefs délais. En fait, privatisation de la terre signifie restitution de la terre. Les moyens choisis pour ce processus ont été la transformation des fermes collectives, par restitution aux anciens propriétaires, et la privatisation des fermes d'État. En Bulgarie, il n'y a pas eu de nationalisation des propriétés foncières après la Seconde Guerre mondiale. Se cotoyaient des fermes collectives et privées, bien que la superficie pouvant être propriété privée fusse limitée. L'idée qui a soutendu la loi de restitution fut le retour sans limite des terrains à leurs propriétaires. À la fin de 1994, en 2 ans et demi, environ $80 \%$ des terres agricoles ont été légalement certifiées en tant que propriétés privées. À la fin de 1993, plus de 1000 nouvelles coopératives agricoles étaient créées ${ }^{6}$.

On peut estimer positif le résultat de la privatisation des terres tant au niveau de la durée qu'à celui des portions de terre privatisée. Mais, on doit intégrer dans le résultat économique : la destruction de l'économie rurale ; la chute de la production agricole ; la part importante de la population rurale indigente, notamment les minorités (tsiganes et turques) ; l'échec du retour de l'exode rural qui s'est déroulé à la fin des années 1960.

\section{Privatisation des entreprises publiques nationales et municipales}

18 Elle s'est déroulée selon la loi entrée en vigueur en 1992, mais une année a été nécessaire pour élaborer les procédures législatives accompagnant le processus de privatisation. Le cours de la privatisation reflète le montant des offres et demandes concernant les entreprises publiques étatiques et municipales. Pour ce qui est de ce processus, la Bulgarie a adopté une réglementation plutôt libérale : il n'y a pas de limite quant au nombre d'entreprises en vente; ce sont les municipalités qui décident de ce qu'elles mettent en vente; peuvent même être vendus des éléments n'étant pas inclus dans le programme annuel de privatisation; les acheteurs étrangers et les acheteurs nationaux bénéficient du même traitement. Plus de $80 \%$ des transactions ont été réalisées aux enchères entre au moins deux candidats. Toutefois, en ce qui concerne la vente aux étrangers, on préfère les petites entreprises ou une participation minoritaire. Théoriquement, le personnel d'une entreprise est perçu comme étant un des principaux agents de la privatisation, mais le nombre de transformations de ce type est faible (5 à la fin mai 1995).

Malheureusement, la privatisation n'est précédée d'aucun recouvrement financier ni de commercialisation. Peu de transactions précisent la place de l'entreprise sur le marché. Une grande part de la privatisation se déroule semi-officieusement, d'une part parce que les managers bulgares sont tenus à l'écart du processus de décisions dans les programmes de privatisation, et, d'autre part, parce que les informations disponibles concernant les entreprises y étant sujettes sont délibéremment limitées. Malgré les affirmations professées, les programmes de privatisation ont été entravés par des intérêts spécifiques, des délibérations et l'inefficacité de l'administration.

20 La plupart des manufactures demeurent encore propriétés publiques, mais elles sont laissées sans direction efficace et sans contrôle. Les problèmes financiers qu'elles connaissent sont dus aux difficultés d'approvisionnement en matières premières et de 
débouchés des produits finis, aux crédits à court terme et aux taxes. La plupart de ces entreprises ont contracté des emprunts qu'elles ne peuvent rembourser.

L'émission d'obligations à long-terme (sous la loi de la "mauvaise dette") et d'obligations de la dette extérieure du pays, avec la possibilité de payer en levs bulgares, aussi bien qu'en monnaie étrangère, ont constitué un véritable pas en avant dans la stimulation du processus de privatisation.

Ainsi, 450 privatisations ont été réalisées, 97 entreprises nationales et 353 entreprises municipales. En 1994, le revenu des privatisations était 11 fois plus important qu'en 1993. À la fin janvier 1995, l'Agence de privatisation avait vendu 35 entreprises sur une liste de 204.

$80 \%$ des revenus totaux de la privatisation servent à financer le Fonds d'État de Reconstruction et du Développement (FERD - 53\%), le Fonds de recouvrement des dépenses de privatisation de entreprises d'État (7\%), le Fonds de Protection de l'Environnement (FPE - $5 \%$ ), et le Fonds pour le développement de l'agriculture (15\%).

Les principales techniques de privatisation utilisées en Bulgarie ont été : la vente aux enchères, l'offre réelle, la vente publique des parts, le leasing de 25 ans avec une clause de rachat, la vente avec transfert du droit de propriété.

Les spécialistes ont soulevé la question de la justification de la privatisation du capital, en mettant en évidence qu'une distribution de la propriété publique sans charge serait plus efficace à long terme. Le principal argument est que les revenus sont répartis entre les banques créditrices locales et étrangères (les obligations internes et externes) et l'État (FERD). Par conséquent, il est difficile de déterminer les résultats fiscaux immédiats de la privatisation sur la part publique. De plus, du fait du faible contrôle post-privatisation, le public a adopté une attitude indifférente, sinon négative, envers la privatisation. Quant à l'effet de la privatisation sur l'emploi, aucune donnée exploitable sur cette question n'existe ${ }^{7}$.

Pour résumer, il est difficile de fournir une définition précise du type de privatisation en Bulgarie, parce que quatre gouvernements se sont déjà succédés sans élaborer de stratégie de privatisation. L'objectif du gouvernement de l'Union des Forces Démocratiques (UFD) était de promulguer la Loi sur la restitution et de supprimer l'idéologie et la pratique de la ferme collective. Les deux gouvernements qui lui ont succédé souhaitaient accomplir la privatisation des terres et débuter la privatisation du capital, vendre la propriété étatique.

\section{Résultats du processus de privatisation dans la Bulgarie post-communiste}

27 Aujourd'hui en Bulgarie, aucun groupe social ou politique ne se prononcerait contre la privatisation. Cependant, un grand nombre de groupes d'intérêt et sociaux prône une logique et une direction du processus de privatisation en contradiction avec ses objectifs et ses intérêts.

28 La volonté de dénationaliser le passif de la direction publique, pour aussi bonne qu'elle soit, se réalise par l'étranglement des entreprises publiques, qui ont été obligées de faire des emprunts à des taux défavorables qu'elles n'ont pu rembourser, provoquant leur décapitalisation ${ }^{8}$. On a mis fin non seulement aux approches innovantes, mais aussi 
au processus normal de la reproduction. La plupart des relations de marché, intérieur et extérieur, ont été détruites. Une fois le transfert effectué, les nouveaux acquéreurs recoivent une structure économique dévaluée, c'est-à-dire facile à obtenir, mais en même temps à moitié détruite.

La finalité économique est effectuée sur le papier. La propriété est transférée de la bureaucratie d'État au secteur privé. Mais, en réalité, cette finalité n'est aucunement accomplie, parce que la structure et le milieu économiques sont à moitié détruits. Ceci est plus vrai pour l'industrie, surtout lourde, que pour les transports'. Actuellement, le problème le plus important pour l'économie bulgare est la croissance de la dette intérieure. En 1995, alors que l'inflation était de $32 \%$, la dette intérieure avait augmenté de $405 \%{ }^{10}$. L'augmentation de la dette intérieure est le résultat d'une politique ad hoc (l'embargo sur la Yougoslavie a gravement nuit à la Bulgarie). Toutefois, la raison principale est la déstabilisation des entreprises publiques engendrée par leurs relations désavantageuses avec les entreprises privées.

Le fondement de l'opposition de l'opinion publique à ce faux modèle de privatisation est l'héritage des attitudes de l'ancien régime sur la "propriété populaire", une propriété publique non divisible ayant besoin d'universalisme et de participation dans sa direction ${ }^{11}$.

\section{Privatisation et légitimation de la propriété}

31 D'un point de vue légal, la Bulgarie dispose d'une législation claire en ce qui concerne la légitimation de la propriété privée. Néanmoins, d'un point de vue politique et éthique, certaines catégories de propriétaires sont publiquement mises en doute. Cependant, les processus de réaction n'ont pas été suffisamment étudiés.

Après 1989, la première catégorie a être critiquée fut la propriété privée de l'ancienne nomenklatura. La législation aboutit à une certaine démoralisation, et également une répression, de ces propriétaires ${ }^{12}$. Certains actes législatifs, notamment deux lois édictées en 1992, mettent en doute la légitimité de l'acquisition (ce qui entre en jeu est la croyance dans la non-rétribution de l'acquisition de cette propriété).

La deuxième catégorie de la controverse éthique et politique est celle de la restitution de propriété. Si la majorité des partis de gauche reconnait la validité de la restitution, la plupart de ses membres doute de sa moralité. On considère les revenus perçus de la restitution comme un gain dépourvu d'assise éthique.

34 La troisième catégorie est le capital commercial. Là encore, les partis de gauche se montrent mécontents de son taux d'accumulation élevé. Les notions classiques de "capital productif" et "capital de spéculation" sont ici sollicitées. De surcroît, certains doutent que la propriété commerciale soit le produit du pillage des producteurs, dans le cas d'entreprises locales, et que les hommes d'affaires violent massivement les règles commerciales ou qu'ils suppriment les producteurs locaux en leur fournissant des biens d'exportation de basse qualité ${ }^{13}$.

35 Le secteur privé est la propriété des managers d'entreprises publiques qui ont contourné la loi et ont développé des organisations économiques privées avec des membres de leur famille ou des amis, imitant et remplaçant, la plupart du temps, les activités des entreprises publiques qu'ils dirigent. Ils forment la quatrième catégorie vivement critiquée. 
On peut donc en conclure que la Bulgarie est victime d'un conflit social aigu, lié au rejet par la population des réalités factuelles et légales.

Toutefois, la privatisation, notamment sous la forme de la distribution de propriété étatique sans intérêt, est bien mieux tolérée que les procédures de privatisation argentcomptant. Elle devrait, par conséquent, servir d'instrument pour assourdir et résoudre les tensions sociales existantes.

\section{Différenciation sociale}

La brutalité relative de la transition politique a stimulé des processus qui ont diversifié la population.

La propriété, en particulier les grandes propriétés, soulève la question des privilèges. Les raisons de sa réalisation importent peu, ce peut être une meilleure gestion économique, un meilleur maniement des lois, une meilleure éducation, etc. La privatisation est un processus de légitimation et de construction du groupe social du contracteur, habituellement caractérisé par des tendances à l'oligarchie. La privatisation aboutit à de nouvelles hiérarchies sociales, de nouvelles "classes supérieures". Selon des opposants bulgares ${ }^{14}$, à la fin de la première phase du processus de privatisation en Bulgarie, il y aura 30 à 40 personnes (familles) qui domineront l'économie. L'histoire bulgare n'aura pas vu, ces 120 dernières années, une telle concentration.

Le modèle de la transition économique imposé génère des élites politiques et économiques. En général, les nouvelles élites ont connu une évolution similaire. La nouvelle configuration politique bulgare est caractérisée par la formation d'un groupenoyau, recherchant une domination durable. Un nouveau type d'union entre pouvoir politique et économique apparait. Sous l'ancien régime, une certaine forme de fusion existait dans le mécanisme de l'État-parti, cette association prend désormais de nouvelles dimensions.

41 Il est devenu évident que le principal motif politique des représentants de l'élite politique n'était pas seulement de "posséder la machine étatique". Leur principal objectif était de capturer le pouvoir étatique, essentiellement comme un moyen d'accéder au pouvoir économique et à une participation effective au processus de privatisation. Un tel point de vue peut paraître une simplification à outrance, mais nous le mettons en avant.

\section{Le processus d'oligarchisation}

La déficience du modèle, choisi ou imposé, de changements sociaux est incontestable pour la plupart des sujets politiques. Trois facteurs auraient pu la prévenir : l'existence d'une opinion publique bien définie; une législation stable; un contrôle étatique effectif.

Si la pensée politique des Bulgares en 1990-1992 n'avait pas été troublée par la confrontation politique et l'arrogance sociale des candidats, si le nihilisme légalisé de 1992 n'avait pas atteint le niveau de 1950 (période stalinienne), ou si le système de contrôle n'avait pas été détruit en tant que symbole de l'immoralité totalitaire, la Bulgarie aurait eu la chance de connaître un développement "européen", socialement 
équilibré, une société sensible offrant la perspective d'une vie décente aux deux-tiers de sa population. Entre 1991 et 1992, la société bulgare a, sinon définitivement, du moins pour une longue période, perdu cette voie possible.

En fait, en raison de l'inertie sociale, la Bulgarie n'a pas choisi le modèle européen de développement malgré l'opinion favorable de la majoritéé ${ }^{15}$ Au lieu d'une "européanisation", on peut dire que la Bulgarie traverse un processus de "latinoaméricanisation". Bien que ne possédant pas ses traits principaux (le pays n'est pas en situation de guerre civile, l'armée n'a aucune ambition politique, et le président semble bien plus présentable que nombre de ses homologues sud-américains), des processus caractéristiques du Tiers-monde sous-développé, périphérique, transforment la société bulgare, comme, par exemple, une différenciation sociale aigue, une corruption généralisée, l'imitation d'un système bancaire, des formes illégales de transformation de la propriété étatique, la création de cercles restreints cumulant pouvoirs politique et économique, contrôlant aussi bien les flux financiers que l'opinion publique.

Le processus de recapitalisation en Bulgarie a débuté en 1990. Il a inévitablement engendré des tendances et des processus que l'on peut qualifier d'"oligarchisation". Bien que la formation d'une oligarchie soit synonyme d'élitisme, durant la période de transformation politique en Bulgarie après 1989, ce processus était considéré antiélitiste par essence.

\section{La nouvelle oligarchie}

Comment pourrions définir le noyau de la nouvelle oligarchie en Bulgarie? Est-ce le simple processus de "production" de nouvelles élites ou sont-ce ses ressources qui expliquent l'oligarchisation?

Le $\mathrm{XX}^{\text {ème }}$ siècle a développé un large éventail d'intelligence et d'analyses du rôle des élites politiques ${ }^{16}$. L'oligarchisation représente un développement déformé des élites, car elle viole le principe le plus important de l'élite moderne : l'auto-justification. Au contraire des élites féodales et aristocratiques du siècle dernier, les nouvelles élites ont réalisé leur initiative sociale en atteignant un statut social supérieur. Au contraire, l'élite oligarchique se repose sur son simple statut et non sur l'initiative.

Dans les sociétés modernes, on compte de nombreux groupes d'élites, politique, économique, mais aussi culturel et religieux. L'unification de l'élite économique et politique, sa réduction à un corps social uni, mais pas totalement homogène, est une affection de la société moderne. L'élite qui se développe sous la forme d'une oligarchie perd ses capacités créatrices, elle forme un parasite vis-à-vis de l'État et de la société, elle est incapable de remplir ses fonctions de dirigeant collectif.

L'oligarchie n'appartient à aucun parti politique. Elle dépersonnalise même les partis et les organisations civiles. Il n'est pas aisé de dire jusqu'à quel point ceci est correct pour la Bulgarie. Mais les banques, les cercles financiers et leurs partisans ont participé à tous les gouvernements bulgares depuis les changements opérés en $1989^{17}$. Le problème n'est pas dans l'union des pouvoirs politique et économique, mais dans celle des propriétés acquises illégalement et légalement.

Le résultat est que les premières sont légitimées et les secondes sans fondement moral. La genèse de l'oligarchie bulgare est une face de la génèse du capital bulgare dans son ensemble, puisqu'elle se nourrit des ressources du capital étatique accumulé. 
51 Le processus d'oligarchisation des nouvelles élites se caractérise par quatre éléments. D'une part, les élites ont intégré les hautes sphères de l'administration et de l'État. D'autre part, les élites maintiennent des liens étroits et synchronisent leurs mouvements avec les cercles bancaires. Troisièmement, les élites ont subi une différenciation sociale, politique et culturelle (particularisation) ${ }^{18}$. Enfin, elles tendent à perpétuer la pyramide sociale établie entre 1990 et 1996.

Pour les cercles en faveur de l'oligarchie, le pouvoir étatique est l'instrument principal de la reconnaissance économique. Les lois édictées afin de distancier le pouvoir de la propriété semblent être inefficaces. Les restrictions imposées aux banques, leur interdisant d'acquérir des propriétés étatiques ne peuvent pas modifier radicalement le rôle des banques en tant qu'instrument de l'oligarchisation des élites.

Il existe, en bulgare, un terme définissant les groupes oligarchiques: "groupements économiques". Les grands groupements économiques bulgares, d'abord sept, puis treize, sont apparus sur la scène publique trois ans auparavant (en 1993). Il est devenu évident, peu après, qu'ils étaient en relation les uns avec les autres et qu'ils disposaient de ressources douteuses quant à l'origine de leurs finances.

La terminologie de groupement économique peut générer une fausse notion d'une union de large envergure de personnes et de capital, dont l'origine est propre. Or la vérité est toute autre. Les grands propriétaires dans les groupements sont peu nombreux et n'apparaissent jamais en public.

La différenciation sociale et culturelle des élites économiques se déroule bien plus rapidement que dans les autres pays européens. L'homme d'affaires bulgare typique "prospère" loue les services d'un garde du corps, bien que son revenu soit de 20 à 50 fois inférieur à celui de son coreligionnaire ouest-européen ayant recours à ce genre de service. Nous voyons ici une autre illustration de la conscience de soi des grands propriétaires, qui sont incertains de la légalité des fondements de leurs propriétés.

56 La tendance à l'oligarchisation des groupements économiques de haut niveau est accentuée par leur tentative de dicter les politiques législatives et gouvernementales. Dans les premières années suivant les changements démocratiques, ils cherchaient à obtenir la reconnaissance politique et s'identifiaient au nouveau parti dénommé "parti des affaires". Aujourd'hui, ils cherchent d'autres moyens plus fiables et immédiats pour la participation au contrôle des pouvoirs, utilisant les deux principaux partis en Bulgarie, le Parti socialiste et l'UFD.

57 L'oligarchie engendre de nouveaux conflits dans la Bulgarie post-communiste. Par exemple, celui entre les intérêts nationaux d'ouverture des marchés bulgares et l'opposition "camouflée" des groupements capitalistes locaux ne désirant pas partager le marché avec des concurrents étrangers. Le nombre de fois où la position des syndicats a coïncidé avec celle des grands détenteurs de capital est remarquable, tandis que les hommes d'affaires de moyenne envergure et les fermiers adoptaient des points de vue radicalement différents. Un autre exemple est la situation des grandes banques semi-privatisées artificiellement soutenues par l'État, qui, dans le même temps, leur impose des sanctions pour investissement infructueux et crédits généreusement offerts.

Les pays de l'Europe de l'est doivent se débarasser de l'égalitarisme primaire de l'époque "communiste". Toutefois, ils sont en train de découvrir une nouvelle caste qui semble discréditer les idéaux démocratiques et l'essence de la société ouverte. Le fait 
que seulement $3 \%$ de la société bulgare évalue sa situation économique comme "très bonne" est loin d'indiquer une société démocratique.

Bien que la structure pyramidale de la société communiste ait été remplacée par une diversité de hiérarchies sociales, l'image du sommet de la hiérarchie est devenue de plus en plus visible. Ce qui distingue le sommet, c'est l'argent, et, pour l'instant, l'argent gagné facilement. En Bulgarie, les personnes au sommet ont gagné énormément d'argent, non pas à la suite de nombreuses transactions, mais "par chance". Une telle stratification sociale aigue engendrée par l'émergence soudaine des élites économiques le rend inadapté au monde culturel et de la communication.

\section{Un dé-oligarchisation est-elle possible?}

Le processus d'oligarchisation en Bulgarie post-communiste n'a pas encore atteint sa phase de maturité, mais il est problématique de savoir si on peut y mettre fin.

Le capitalisme périphérique, ainsi que l'apparence sociale et politique de la Bulgarie actuelle est en opposition avec les tentatives d'introduire une législation antioligarchique et, encore plus, avec celles de débuter son application. Il est évident que l'oligarchie ne peut défendre ses positions sans l'aide ni l'existence de loyaux officiels corrompus. À l'heure actuelle, l'État ne dispose d'aucun moyen de l'arrêter.

On ne pourra mettre un frein à la nouvelle oligarchie bulgare qu'à la fin du processus de privatisation et de redistribution. Jusque là, toutes les formes de rejets et d'opposition connaîtront le même sort que les caricatures des journaux.

\section{NOTES}

7. Parvulov (Sv.), « Privatisation in Bulgaria », Report, mars 1995.

8. Vasilev (G.), « Privatisation and Deblockage of Society », Sociological Problems, (4), 1993.

9. Cf. Genov (N.), ed., The Riscs of the Transition, Sofia : National and Global Development, 1994.

10. Tomov (A.), « One Year After the Ruling of the Left », Trud, 06/02/96.

11. Donchev (St.), "Strategies and Behaviour of Political Actors in the Process of Privatisation ", Sociological Problems, (4), 1993.

12. Popov (M), "The Legislation and the Process of Privatization ", Capital, février 1995, pp. 12-13.

13. Chipev (P.), « Market against Mass Privatization », Sociological Problems, (3), 1993, pp. 17-31.

14. Stoichev (K), Krastev (I), "The Ruling of Democratic Left », Sega, 1996, pp. 3-5, 7-16.

15. Levinson (A.), « Macroeconomic Uncertainty in the Market Transition », in Genov (N.), ed., The Riscs of the Transition, Sofia : National and Global Development, 1994.

16. Kanev (D.), «The Future of Bulgarian Democracy », Politological Studies, 1994, pp. 19-22.

17. Draganov (M.), The Social Allergies, Sofia: Ch. Botev, 1994 ; Popov (M.), « Oligarchy and Civil Society ", Novo Vreme, 1996.

18. Todorova (E.), Democratic Attitudes in Bulgaria, Sofia : CINTI, 1996. 


\section{RÉSUMÉS}

L'effet destructeur de la privatisation de la propriété d'État dans la Bulgarie postcommuniste est au cœur de la construction d'une nouvelle stratification et division politique de la société bulgare. Le marché et la privatisation exercent des impacts différents sur les motivations et les intentions politiques. La privatisation légale et illégale ne sont pas encore clairement distinctes. Un nouveau type d'oligarchie est généré, résultat de la conjonction de la privatisation avec une faible réaction dans la conscience civile, une incongruence élévée entre la culture économique et l'expérience de marché des citoyens bulgares, un blocage de l'administration publique qui dure depuis cinq ans maintenant.

Sans de réelle reconstruction de l'économie et une stratégie précise quant aux façons et moyens de redistribuer la richesse nationale sur une échelle plus large, au contraire de ce qui est en vigueur jusqu'à présent, on ne peut que difficilement s'attendre à une prospérité économique pour la Bulgarie.

The destructiveness and privatization of the state ownership in postcommunist Bulgaria are in the core of the building of the new political and stratification map of the Bulgarian society. The market and mass privatization have different impact on the political motives and intentions.

The legal and illegal privatization are not yet fully detached. A new type of oligarchy is generated as a result of conjunction of privatization with low reactivity in the civil consciousness; a high discrepancy in the economic culture and market experience of Bulgarian citizens; blockade of the public administration that has lasted five years now.

Without actual reconstruction of the economy and clear strategy for the ways and means to redistribute the national wealth on a broader civil scale than it has been the practice up to now we hardly could expect a positive economic future for Bulgaria.

\section{AUTEURS}

\section{MIROSLAV K. POPOV}

M. Popov est membre du Comité exécutif du Conseil du Parti socialiste bulgare. Il est également lecteur à l'Université libre de Varna et à la Nouvelle Université bulgare. Parmi ses dernières publications se trouvent : Le gouvernement socialiste et l'opinion publique (Sofia : Ch. Botev, 1995), La gauche au pouvoir après les élections de 1994 (Sofia : BSP, 1996), et La présidence et la lutte politique, 1990-1996 (Sofia : Ch. Botev, 1996).

\section{ELKA N. TODOROVA}

E. Torodov est la directrice du département de sociologie politique de l'Institut de Sociologie de l'Université libre de Varna. Elle a dernièrement publié : Ethnicity and Politics in Bulgaria and Israel, éd. (Avebury, 1993), Social Psychology (Briag, 1995), et Attitudes démocratiqes en Bulgarie (Sofia : Cinti, 1996). 